ESJ Natural/Life/Medical Sciences

\title{
Effets des Demi-Lunes Multifonctionnelles sur la Production du Sorgho en Afrique de l'Ouest: Cas de la Région de Tahoua au Niger
}

\author{
Maman Nassirou Ado
}

Mahamadou Sani Moussa

Faculté des Sciences Agronomiques, Université de Tahoua, Tahoua, Niger

\section{Harouna Karimou Ambouta}

Faculté d’Agronomie, Université Abdou Moumouni, Niamey, Niger

Doi:10.19044/esj.2021.v17n34p112

Submitted: 31 May 2021

Accepted: 16 July 2021

Published: 30 September 2021
Copyright 2021 Author(s)

Under Creative Commons BY-NC-ND 4.0 OPEN ACCESS

Cite As:

Ado M.N, Moussa M.S. \& Karimou Ambouta H. (2021). Effets des Demi-Lunes Multifonctionnelles sur la Production du Sorgho en Afrique de l'Ouest : Cas de la Région de Tahoua au Niger. European Scientific Journal, ESJ, 17(34), 112.

https://doi.org/10.19044/esj.2021.v17n34p112

\section{Résumé}

La dégradation des sols constitue un problème écologique ayant des conséquences néfastes considérables sur la production alimentaire au Niger et dans la région de Tahoua en particulier. L’objectif de cette étude, conduite à Kalfou dans la région de Tahoua au Niger, est d'évaluer les effets des demilunes multifonctionnelles sur la production agricole de sorgho. Ainsi l'essai expérimental, installé sur un terrain initialement encrouté et dénudé pendant deux (2) campagnes agricoles successives, est un bloc randomisé complet composé de trois (3) traitements sur (i) un sol aménagé en zaï (Zaï), (ii) un sol aménagé en demi-lunes conventionnelles (DLC) et (iii) un sol aménagé en demi-lunes multifonctionnelles intégrant à la fois les zaï et les tranchés de reboisement (DLM). Les résultats de l'étude ont montré que la croissance des cultures du sorgho est significativement plus élevée dans les ouvrages de demi-lunes multifonctionnelles que dans les ouvrages de zaï et de demi-lunes conventionnelles. En outre, les ouvrages de demi-lunes multifonctionnelles ont permis de produire 639 et $2159 \mathrm{Kg} / \mathrm{ha}$ de grains de sorgho respectivement en première et deuxième campagne. Ce rendement en grains est 3 à 4 fois plus élevé dans les ouvrages de demi-lunes multifonctionnelles que dans les ouvrages de zai et des demi-lunes conventionnelles. Ainsi, l'introduction de 
ces nouveaux ouvrages de demi-lunes multifonctionnelles, adaptés au contexte de la région sahélienne de Tahoua au Niger, permet d'optimiser l'utilisation des éléments nutritifs et des eaux de ruissellement pour la production agricole, ce qui peut renforcer la résilience de la population dans ladite zone. Toutefois, il serait nécessaire d'évaluer les impacts de ces ouvrages sur la production agricole à long terme tout en intégrant également d'autres cultures.

Mots clés : Dégradation Des Sols, Restauration Des Terres, Zaï, Demi-Lunes Conventionnelles, Demi-Lunes Multifonctionnelles, Agriculture, Tahoua, Niger, Afrique De l'Ouest

\title{
Effects of Multifunctional Half-Moons on Production of Sorgho in West Africa: The Case Study of Tahoua Region in Niger
}

\author{
Maman Nassirou Ado \\ Mahamadou Sani Moussa
}

Faculté des Sciences Agronomiques, Université de Tahoua, Tahoua, Niger

Harouna Karimou Ambouta

Faculté d’Agronomie, Université Abdou Moumouni, Niamey, Niger

\begin{abstract}
Soil degradation is an ecological problem which has considerable negative consequences on food production in Sahel. The objective of this study, undertaken in Kalfou in the Tahoua region in Niger, was to test the effectiveness of multifunctional half-moons (DLM) for agricultural production of sorghum. The experiment design, installed on soil encrusted two (2) successive cropping seasons of sorghum, was a complete randomized block composed of three (3) treatments in three (3) : i) soil with local practice of zai, ii) soil with conventional half-moons (DLC) and iii) soil with multifunctional half-moons (DLM). Results showed that sorghum crop growth was significantly higher in multifunctional half-moons than in zaï and conventional half-moons structures. Besides,, the multifunctional half-moon structures produced 639 and $2159 \mathrm{~kg}$.ha-1 of sorghum grain at the first and second season respectively. This grain yield was three (3) to four (4) times higher in multifunctional half-moon structures than in zai and conventional half-moon structures. Thus, the introduction of these new multifunctional halfmoon structures, adapted to the context of Tahoua region allows optimizing the use of nutrients and runoff water for agricultural production, which can improve people's resilience in the zone. Nevertheless, it would be necessary
\end{abstract}


to evaluate the impacts of these rain water harvesting technologies on agricultural production in the long-term while also integrating other crops.

Keywords: Soil Degradation, Land Restoration, Zaï, Conventional HalfMoons, Multifunctional Half-Moons, Agricultural Production Of Sorghum

\section{Introduction}

En Afrique, l'exploitation des ressources en terres pour l'agriculture est la principale source alimentaire de la population. Cette activité est pratiquée essentiellement par des petits exploitants en culture pluviale. On estime qu'en Afrique subsaharienne, 95\% des terres cultivées sont sous l'agriculture pluviale (Svendsen et al., 2009). Cependant, dans cette zone l'agriculture est tributaire des aléas climatiques et des conditions pédologiques très souvent défavorables (Sawadogo, 2006 ; Roose, 2017). En effet, 65 \% des terres en Afrique sont dégradées et le continent voit disparaître chaque année pratiquement trois (3) millions d'hectares de forêts et les pertes de terres et de nutriments amputent son PIB annuel de 3 \% (Banque Mondiale, 2016). Ainsi, les agriculteurs dépendent de plus en plus de terres marginales pour la production alimentaire en raison de la dégradation continue des sols, de la disponibilité limitée des terres fertiles et de la pression démographique croissante (il serait mieux de mettre la source). Ces terres dégradées fournissent généralement des rendements médiocres (Wildemeersh et al., 2015 ; Roose, 2017). Par ailleurs, la perte d'eau de pluie par des voies non productives notamment le ruissellement limite sérieusement l'agriculture pluviale en Afrique subsaharienne (Biazin et al., 2012). On estime qu'au Sahel dont le Niger, seulement une très petite fraction $(<10 \%)$ de l'eau de pluie qui est utilisée par les cultures La majeure partie de ces eaux de pluie est perdue sous forme d'eau d'évaporation (30-50\%), de ruissellement (25-50\%) et de percolation profonde (10-30\%) (Rockström et al., 1998 ; Wildemeersh et al., 2015). Cette situation a eu des conséquences néfastes sur l'environnement et a entraîné, de ce fait, un retard dans le décollage économique de cette zone d'Afrique (Ambouta et Bouzou, 2004 ; Roose, 2004). Dans un tel contexte, la réhabilitation des terres dégradées pour l'amélioration de la productivité des terres par les techniques de conservation des eaux et des sols (C.E.S) est une alternative afin de restaurer la productivité des terres et assurer les besoins alimentaires de la populatio Il s'agit non seulement de réduire le ruissellement et l'érosion, mais de recouvrir la terre, de soigner le sol et de nourrir les plantes pour la production agricole (Roose et al., 2010). Plusieurs études ont montré l'efficacité de ces techniques à restaurer la productivité des terres dégradées (Biazin et al., 2012; Zougmoré et al., 2014; Roose, 2017; Yosef et Asmamaw, 2015). Récemment, des études ont également confirmé que les ouvrages de restauration des terres permettent d'augmenter l'humidité du sol, la 
disponibilité des nutriments et de favoriser la croissance des plantes cultivées (Wildemeersh et al., 2015; Ding et al., 2021; Tadros et al., 2021) afin d'augmenter la production agricole et d'améliorer la sécurité alimentaire de la population (Nyamekye et al., 2018; Kokerai et Kagedera, 2019).

Ainsi, ces ouvrages de restauration des terres ont fait l'objet de diffusion chez les producteurs notamment au Niger. Dans ce pays, il a été estimé que 1,2 million d'agriculteurs ont restauré 5 millions d'hectares de terres arables et planté 200 millions d'arbres grâce à leurs propres ressources (Banque Mondiale, 2016). Cependant, des études récentes ont montré que le taux d'adoption des techniques de récupération de terres dégradées reste encore faible car les rendements des cultures qui y sont pratiquées (mil, niébé) ne justifient pas les investissements en termes de main d'œuvre et de matériel requis par ces techniques (Wildemeersh et al., 2015). Dans les perceptives d'améliorer l'efficacité de ces ouvrages de restauration des terres dégradées, des innovations sont de plus en plus initiées. C'est le cas notamment des demilunes multifonctionnelles, intégrant à la fois les zai et les tranchées de reboisement, introduites au Niger particulièrement dans la région sahélienne de Tahoua où la pluviométrie est faible et aléatoire. Les principaux objectifs visés à travers ces nouveaux ouvrages sont d'optimiser l'utilisation de l'eau et des éléments nutritifs et de diversifier la production agro-sylvo-pastorale. Cependant, le fonctionnement et la valeur ajoutée de ces nouveaux ouvrages par rapport aux ouvrages conventionnels ne sont pas encore scientifiquement prouvés surtout dans un contexte d'irrégularité des pluies à l'image de la région de Tahoua au Niger. L'objectif de cette étude est de tester le fonctionnement et d'évaluer les effets des demi-lunes multifonctionnelles sur la production agricole par comparaison avec les pratiques locales et les ouvrages conventionnels.

\section{Matériel et Méthodes}

\subsection{Site d'étude}

L'étude a été conduite à Kalfou (14\%52.263' latitude Nord et 005³1.067' longitude Est) qui est situé à 35 km au Nord-est de la ville de Tahoua au Niger (Figure 1). Le climat de la zone est de type sahélien avec une moyenne pluviométrique annuelle durant les dix dernières années estimée à $305 \mathrm{~mm}$. Le site expérimental est un terrain abandonné sur un plateau d'une pente de 2 à $3 \%$ et est caractérisé par un sol dénudé, encrouté suite aux processus de ruissellement et de l'érosion en nappe. Ce qui empêche l'exploitation agricole de ce terrain. 


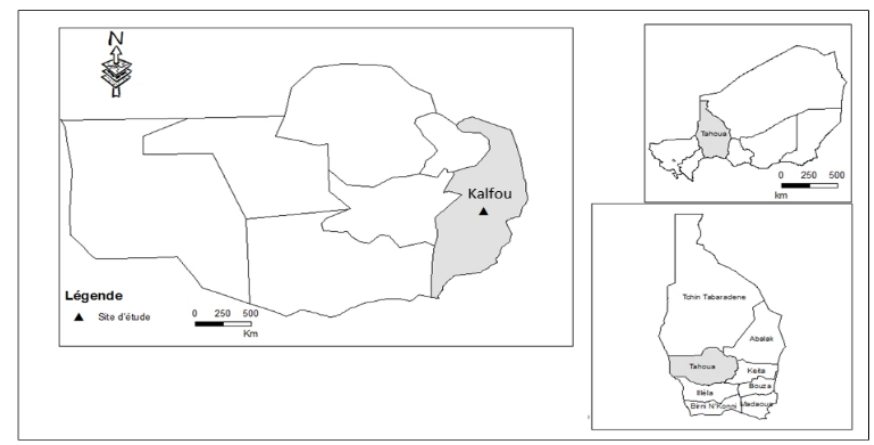

Figure 1. Localisation du site d'étude

\subsection{Matériel végétal}

Le matériel végétal utilisé pour la conduite de l'étude est composé essentiellement de la variété de sorgho Mota-Maradi, provenant du CERRA de Maradi au Niger qui a un cycle de 75 à 80 jours et un rendement potentiel de $2000 \mathrm{~kg} / \mathrm{ha}$ (MA, 2012).

\subsection{Dispositif expérimental}

Le dispositif expérimental est un bloc randomisé ( 0.5 ha) complet composé de trois(3) traitements en trois(3) répétitions sur des parcelles élémentaires de $15 \mathrm{~m}$ x $15 \mathrm{~m}$ espacées les unes des autres de $2 \mathrm{~m}$ (Figure 1) sur un sol aménagé en zaï considéré comme témoin en pratique locale (Zaï), (ii) un sol aménagé en demi-lunes conventionnelles (DLC) et sur (iii) un sol aménagé en demi-lunes multifonctionnelles intégrant à la fois les zaï et les tranchés de reboisement (DLM) (Figure 2).

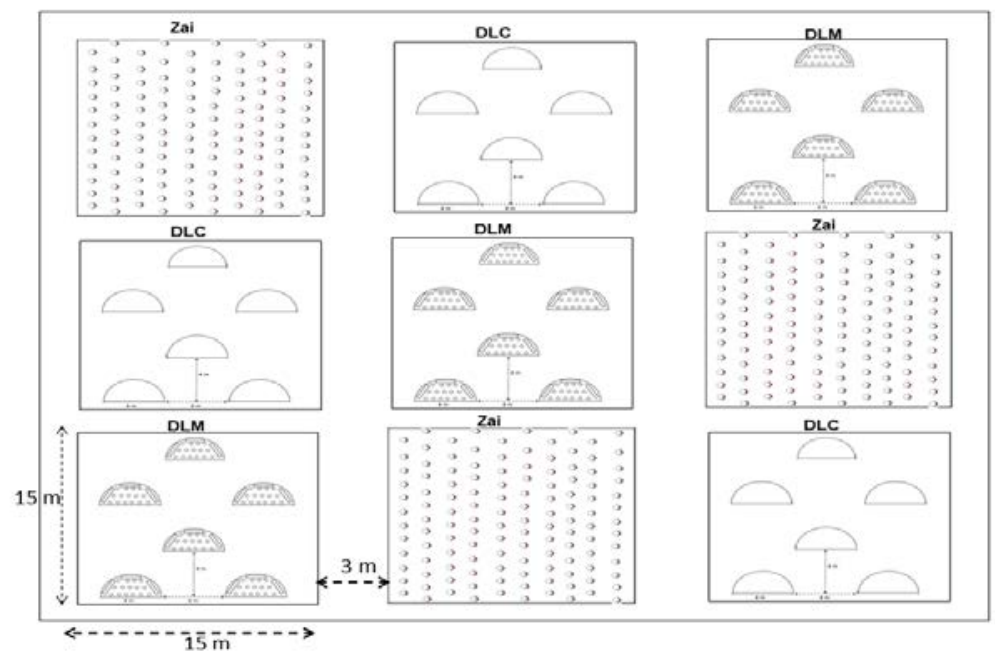

Figure 2. Schéma du dispositif expérimental de l'essai 
Zai sont des parcelles aménagées en zaï, DLC sont des parcelles aménagées en demi-lunes conventionnelles et DLM sont des parcelles aménagées en demi-lunes multifonctionnelles.

Les Zaï, ouvrages de $30 \mathrm{~cm}$ de diamètre et $20 \mathrm{~cm}$ de profondeur, ont été installés en quinconce perpendiculairement à la pente selon des écartements de $70 \mathrm{~cm}$ et $100 \mathrm{~cm}$ respectivement sur et entre les lignes (Figure 3). Les DLC, ouvrages de $4 \mathrm{~m}$ de diamètre et $20 \mathrm{~cm}$ de profondeur d'excavation, ont été installés perpendiculairement à la pente selon un écartement de $4 \mathrm{~m}$ sur la même ligne et entre deux (2) lignes voisines (Figure 3). La terre de déblais est déposée sur le demi-cercle en un bourrelet semicirculaire au sommet aplati. Les DLM, installées selon le même dispositif que les DLC sans excavation à l'intérieur, intègrent 16 zaï disposés en quinconce et 3 tranchés de reboisement de $1 \mathrm{~m}$ de longueur et $50 \mathrm{~cm}$ de largeur et $50 \mathrm{~cm}$ de profondeur (Figure 3).
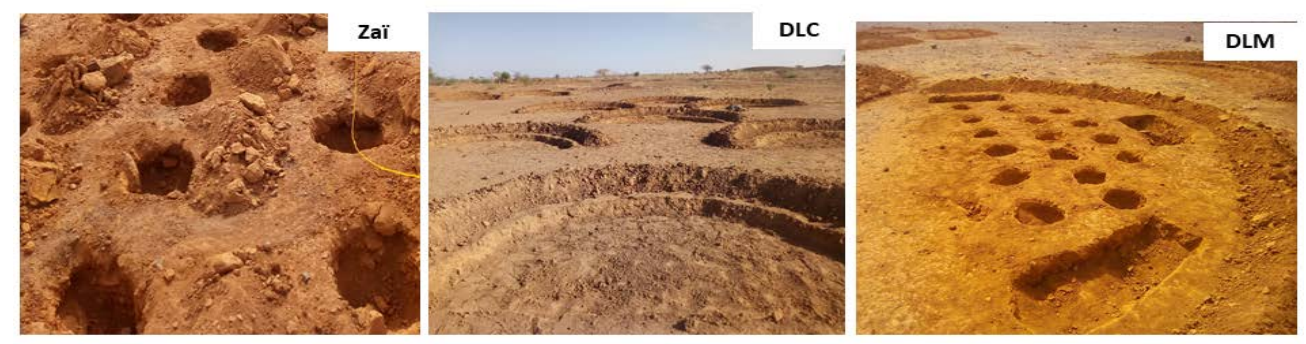

Figure 3. Photographies des différents types d'ouvrages sur le site de l'essai.

Zai sont des parcelles aménagées en zaï, DLC sont des parcelles aménagées en demi-lunes conventionnelles et DLM sont des parcelles aménagées en demi-lunes multifonctionnelles.

A l'installation du dispositif, 5 échantillons du sol ont été prélevés respectivement sur les 4 côtés et au milieu de la grande parcelle d'essai (soit un échantillonnage en X). Les échantillons prélevés ont été analysés au Laboratoire des Sciences du Sol de la Faculté d’Agronomie de l'Université Abdou Moumouni de Niamey (Niger) pour déterminer les caractéristiques physico-chimiques du sol à l'état initial.

\subsection{Conduite de l'essai}

L'essai a été conduit pendant deux campagnes de cultures de sorgho respectivement en 2019 et 2020 pendant les saisons des pluies au Niger.

Après l'installation des ouvrages et avant le semi de la première campagne, le compost à base du fumier des petits ruminants a été appliqué uniformément dans les ouvrages selon une dose de $10 \mathrm{t} / \mathrm{ha}$ soit $80 \mathrm{~g}$ par ouvrage de zai et $7 \mathrm{~kg}$ par ouvrage de demi-lune. Les mesures et les observations ont été réalisées sur les paramètres de croissance notamment le 
nombre de talles, la hauteur des plants, le diamètre des tiges chaque semaine pendant les saisons culturales. Les rendements en biomasse et en grains de sorgho ont été évalués sur les différents traitements à la fin de chaque campagne agricole .

\subsection{Traitement statistique des données}

Les analyses de variance (ANOVA) ont été réalisées sur les variables agronomiques de sorgho notamment les paramètres de croissance et les rendements de sorgho en utilisant le logiciel XLSTAT version 2016. Le test de Fisher au seuil de 5\% a été utilisé pour séparer les moyennes deux à deux.

\section{Résultats et Discussion}

\subsection{Résultats}

\subsubsection{Caractéristiques des sols sur le site expérimental}

Le sol du site expérimental à l'état est de type ferruginosol caractérisé par une texture sablo-argileuse avec un $\mathrm{pH}$ acide. Il est marqué par des faibles teneurs en matières organiques, en bases échangeables et en phosphore assimilable (Tableau 1).

Tableau 1. Caractéristiques physico-chimiques du sol du site à l'état initial

\begin{tabular}{|c|c|c|c|c|c|c|c|c|c|c|c|c|c|}
\hline $\mathrm{pH}$ & \multicolumn{5}{|c|}{ Bases échangeables (meg/100g) } & $\mathrm{P}(\mathrm{ppm})$ & \multicolumn{4}{|c|}{ matières organiques (\%) } & \multicolumn{3}{|c|}{ Granulométrie (\%) } \\
\hline $\mathrm{H}_{2} \mathrm{O}$ & $\begin{array}{c}\mathrm{Ca}^{+} \\
+\end{array}$ & $\mathrm{Mg}^{++}$ & $\mathrm{Na}^{+}$ & $\mathrm{K}^{+}$ & CEC & Assimilable & $\mathrm{C}$ & M.O & $\mathrm{N}$ & $\begin{array}{l}\mathrm{C} / \\
\mathrm{N}\end{array}$ & Argiles & Limons & Sables \\
\hline 5,1 & 3,5 & 0,6 & 0,2 & 0,3 & 4,7 & 16,1 & 0,2 & 0,4 & 0,03 & 6,7 & 32,3 & 13,8 & 53,9 \\
\hline
\end{tabular}

\subsubsection{Evolution de la hauteur des plants}

La figure 4 présente l'évolution des hauteurs des plants du sorgho au niveau des différents traitements pendant les deux campagnes culturales. Il ressort de cette figure que la hauteur des plants du sorgho augmente avec le temps pour tous les traitements pour les deux campagnes. La hauteur maximale des plants est comprise entre 120 et $170 \mathrm{~cm}$ et entre 195 et $215 \mathrm{~cm}$ respectivement pendant la première et la deuxième campagne culturale. Ainsi, la hauteur des plants est plus élevée pendant la campagne de 2020 que pendant celle de 2019. La hauteur des plants est plus élevée à toutes les dates au niveau de DLM qu'au niveau de DLC et zai où une évolution similaire des plants de sorgho est observée.

A la fin du stade de montaison avant l'épiaison, correspondant aux $71^{\text {ème }}$ et $68^{\text {ème }}$ jours après le semi respectivement pendant la première et la deuxième campagne, les plants du sorgho sont significativement plus longs sur le sol aménagé en DLM que sur le sol aménagé en DLC et en zaï. La hauteur des plants est de 168 et $215 \mathrm{~cm}$ respectivement en 2019 et 2020 dans les DLM contre 124 et $196 \mathrm{~cm}$ dans les zaï (Figure 4). 


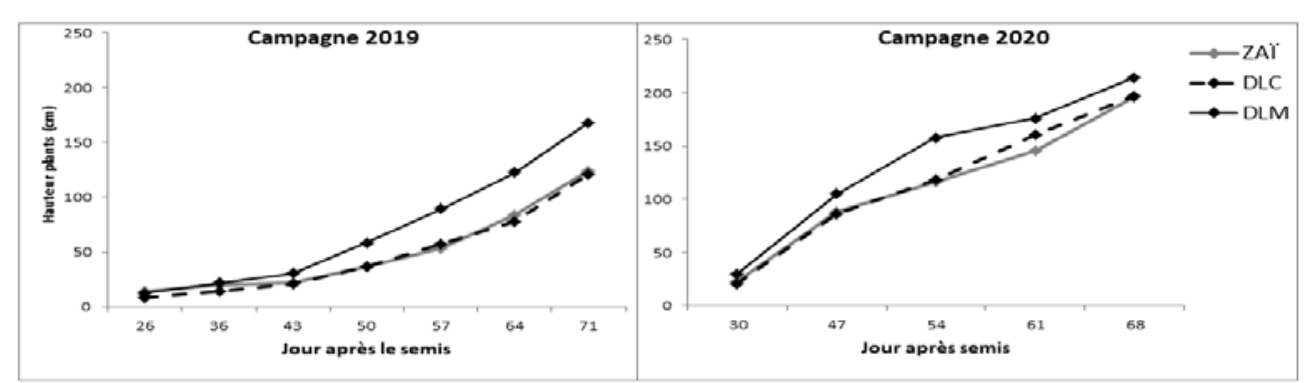

Figure 4. Evolution de hauteur des plants du sorgho au niveau des différents traitements pendant les campagnes 2019 et 2020

Zai est la pratique locale considérée comme témoin, DL est le sol aménagé avec des demi-lunes conventionnelles, DLM est le traitement du sol aménagé avec des demi-lunes multifonctionnelles.

\subsubsection{Nombre des talles et diamètres des plants}

Les résultats du tableau 2 montrent que le nombre des talles et le diamètre des plants sont plus élevés pendant la deuxième campagne que pendant la première campagne au niveau de tous les traitements. Le nombre des talles est compris entre 3 et 5 pendant la première campagne agricole alors qu'il a varié de 7 à 9 pendant la deuxième campagne. Par ailleurs, le nombre des talles et le diamètre des plants sont significativement plus élevés dans les ouvrages DLM que dans les ouvrages de Zai et de DLC pendant les deux campagnes excepté le nombre de talles pendant la première campagne qui est statistiquement similaire entre les différents traitements.

Tableau 2.Variation du nombre des talles et du diamètre des plants en fin stade de montaison selon les traitements

\begin{tabular}{|c|c|c|c|c|}
\hline Campagne & Variables & Zai & DLC & DLM \\
\hline \multirow{2}{*}{2019} & Nombre des talles & $3,2 \mathrm{a}$ & $3,6 \mathrm{a}$ & $4,5 \mathrm{a}$ \\
\cline { 2 - 5 } & Diamètre des plants & $12,6 \mathrm{a}$ & $11,7 \mathrm{a}$ & $15,8 \mathrm{~b}$ \\
\hline \multirow{2}{*}{2020} & & & & \\
& Nombre des talles & $7,3 \mathrm{a}$ & $7,0 \mathrm{a}$ & $8,7 \mathrm{~b}$ \\
\cline { 2 - 5 } & Diamètre des plants $(\mathrm{mm})$ & $14,7 \mathrm{a}$ & $14,9 \mathrm{a}$ & $16,2 \mathrm{~b}$ \\
\hline
\end{tabular}

Zai est la pratique locale considérée comme témoin, DLC est le sol aménagé avec des demi-lunes conventionnelles, DLM est le traitement du sol aménagé avec des demi-lunes multifonctionnelles Les moyennes affectées par les mêmes lettres pour une même variable et par campagne ne sont pas statistiquement différentes selon le test de Fisher au seuil de 5\%.

\subsubsection{Rendements en biomasses de sorgho}

Les biomasses fraiches et sèches du sorgho sont plus élevées pendant la deuxième année que pendant la première année au niveau de tous les 
traitements (Figure 5). La quantité de biomasse fraiche est comprise entre 1840 et $6016 \mathrm{~kg} / \mathrm{ha}$ et entre 3439 et $13455 \mathrm{~kg} / \mathrm{ha}$ respectivement pour la première et deuxième campagne agricole. Quant à la biomasse sèche, sa quantité est comprise entre 1000 et $3350 \mathrm{~kg} / \mathrm{ha}$ et 1800 et $7000 \mathrm{~kg} / \mathrm{ha}$ respectivement pour la première et la deuxième campagne. Les biomasses fraiches et sèches sont significativement plus élevées dans les demi-lunes multifonctionnelles (DLM) que dans les demi-lunes conventionnelles et les zaï pour les deux campagnes. En effet, la biomasse fraiche est 3,3 fois plus élevée dans les DLM (6016 et $13455 \mathrm{~kg} / \mathrm{ha}$ ) que dans les Zai (1840 et 3439 $\mathrm{kg} / \mathrm{ha}$ ) respectivement pendant la première et la deuxième campagne (Figure 5).

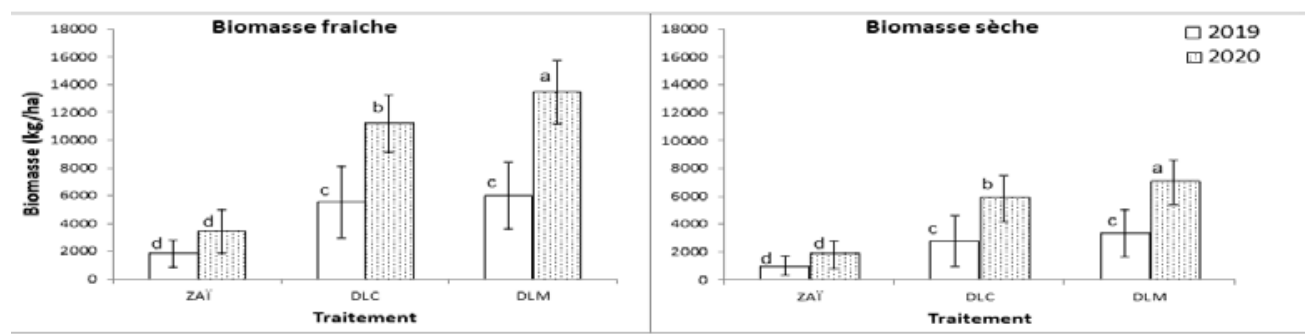

Figure 5. Variations des rendements en biomasses fraiche et sèche du sorgho au niveau des différents traitements par campagne agricole

Zai est la pratique locale considérée comme témoin, DLC est le sol aménagé avec des demi-lunes conventionnelles, DLM est le traitement du sol aménagé avec des demi-lunes multifonctionnelles. Les barres représentent les écarts types des moyennes. Les moyennes présentées en histogrammes affectées par les mêmes lettres pour une même variable ne sont pas statistiquement différentes selon le test de Fisher au seuil de 5\%.

\subsubsection{Rendements en grains de Sorgho}

Le tableau 3 présente le rendement en épis et en grains du sorgho récoltés pendant les deux campagnes agricoles. En effet, le rendement en grains du sorgho est significativement plus élevé à la fin de la deuxième campagne qu'à la fin la première eampagne-au niveau de tous les traitements. Il est compris entre 691 et $2159 \mathrm{~kg} / \mathrm{ha}$ à la fin de la campagne de 2020 soit environ 4 fois plus élevé qu'à la fin de la campagne de 2019 où le rendement est compris entre 188 et $638 \mathrm{~kg} / \mathrm{ha}$. Par ailleurs, le rendement en grains de sorgho est significativement plus élevé dans les ouvrages de demi-lunes multifonctionnelles (DLM) que dans les ouvrages de demi-lunes conventionnelles (DLC) et dans le Zai à la fin de chaque campagne agricole. A la fin de la deuxième campagne, il est 3 fois plus faible dans les ouvrages de Zai (691 kg/ha) que dans les ouvrages de demi-lunes multifonctionnelles (2159 kg/ha) montrant ainsi l'intérêt agronomique de ces ouvrages de DLM 
pour l'amélioration de la production du sorgho dans la région sahélienne de Tahoua, au Niger.

Tableau 3. Rendement en grains de sorgho par traitement

\begin{tabular}{|c|c|c|c|}
\hline \multirow{2}{*}{ Année } & Traitements & Poids des épis (kg/ha) & Rendement grains (kg/ha) \\
\hline \multirow{3}{*}{2019} & ZAI & $273( \pm 182) \mathrm{d}$ & $189( \pm 114) \mathrm{c}$ \\
\cline { 2 - 4 } & DLC & $752( \pm 328) \mathrm{cd}$ & $497( \pm 198) \mathrm{bc}$ \\
\cline { 2 - 4 } & DLM & $1062( \pm 306) \mathrm{c}$ & $638( \pm 180) \mathrm{b}$ \\
\hline \multirow{3}{*}{2020} & ZAI & $1019( \pm 339) \mathrm{c}$ & $691( \pm 231) \mathrm{b}$ \\
\cline { 2 - 4 } & DLC & $2570( \pm 743) \mathrm{b}$ & $1858( \pm 563) \mathrm{a}$ \\
\cline { 2 - 4 } & DLM & $3114( \pm 682) \mathrm{a}$ & $2159( \pm 463) \mathrm{a}$ \\
\hline
\end{tabular}

Zai est la pratique locale considérée comme témoin, DLC est le sol aménagé avec des demi-lunes conventionnelles, DLM est le traitement du sol aménagé avec des demi-lunes multifonctionnelles. Les valeurs entre parenthèses indiquent les écarts types des moyennes.

\section{Discussion}

Tout comme les ouvrages de zaï et des demi-lunes conventionnelles, les nouveaux ouvrages des demi-lunes multifonctionnelles sont opérationnelles dans la région de Tahoua au Niger et permettent la croissance et le développement des cultures du sorgho sur un terrain initialement encrouté et inculte. En effet, pendant deux campagnes agricoles en saisons des pluies au Niger, les cultures du sorgho se sont développées dans les ouvrages pour une hauteur maximale de 168 à 215 cm dans les demi-lunes multifonctionnelles contre 120 à $197 \mathrm{~cm}$ dans de demi-lunes conventionnelles et 124 à $196 \mathrm{~cm}$ dans les Zaï. Ces hauteurs des plantes cultivées du sorgho sont similaires à celles indiquées dans le catalogue national des espèces végétales au Niger pour la même culture (MA, 2012) et à celles rapportées par Abdou et al. (2014). Cette croissance des plantes cultivées du sorgho serait expliquée par l'impact des ouvrages de restauration à augmenter la quantité d'eau stockée dans le profil du sol et réduire le ruissellement des eaux de surface en augmentant le taux d'infiltration de l'eau de pluie, ce qui augmente davantage la disponibilité d'eau dans la zone racinaire des plantes cultivées (Motsi et al., 2004 ; Amede et al., 2011; Kokerai et Kugedera, 2019). En outre, la décomposition du compost apporté dans les ouvrages améliore la structure du sol, favorise l'infiltration et augmente la disponibilité en éléments nutritifs pour les cultures tels que rapportés par plusieurs auteurs (Zougmoré et al., 2003; Sawadogo, 2006; Fatondji et al., 2009; Ganry et Thuriès, 2017).

Par ailleurs, les ouvrages de demi-lunes multifonctionnelles ont permis de produire 639 et $2159 \mathrm{~kg} /$ ha de grains de sorgho respectivement en première (2019) et deuxième (2020) année de campagne. Ce rendement en grains est 4 fois plus élevé dans les ouvrages de demi-lunes multifonctionnelles que dans 
les ouvrages de zai et de demi-lune conventionnelle. Il est de 189 et $691 \mathrm{~kg} / \mathrm{ha}$ dans les ouvrages de zai et 497 et $1858 \mathrm{~kg} / \mathrm{ha}$ dans les demi-lunes conventionnelles respectivement à la fin la première (2019) et la deuxième (2020) campagne. Le rendement en grains obtenu dans les ouvrages de demilunes multifonctionnelles notamment à la fin de la deuxième campagne est comparable à celui indiqué dans le catalogue national des espèces végétales au Niger pour cette variété de sorgho Mota-Maradi (2000 Kg/ha) (MA, 2012). Par contre, le rendement est plus élevé que celui obtenu par Zougmoré et al. (2003) dans les demi-lunes conventionnelles sur des ferralsols lessivés au Burkina Faso (1000kg/ha) ayant reçu du compost (14t/ha). Le rendement en grains de sorgho est significativement plus élevé à la fin de la deuxième année qu'à la fin de la première année contrairement aux résultats obtenus par Zougmoré et al. (2003). Ces résultats seraient expliqués par l'effet de la matière organique issue de la décomposition des résidus des cultures de la première campagne qui aurait amélioré la structure du sol et augmenté la disponibilité en eau et en éléments nutritifs pour les cultures pendant la deuxième campagne (Ganry et Thuriès, 2017). Par ailleurs, les rendements en biomasses et en grains sont significativement plus élevés dans les ouvrages des demi-lunes multifonctionnelles que dans les ouvrages de zai et de demilunes conventionnelles pendant les deux campagnes. En effet, les biomasses (fraiche et sèche) sont 4 fois plus élevées dans les DLM que dans les Zai pendant les deux campagnes. A la fin de la deuxième campagne, le rendement en grains est 3 fois plus faible dans les ouvrages de zai $(691 \mathrm{~kg} / \mathrm{ha})$ que dans les ouvrages de demi-lunes multifonctionnelles (2159 kg/ha). Ces résultats seraient expliqués par la disponibilité en eau dans le sol pour les cultures qui serait plus élevée dans les ouvrages de demi-lunes multifonctionnelles que dans les autres ouvrages. En effet, les ouvrages demi lunes multifonctionnelles semblent être plus favorables à optimaliser l'utilisation des nutriments et des eaux de ruissellement collectées par les cultures par rapport aux ouvrages de zai qui ont un impluvium plus petit que celui de demi-lune et de DLC où l'eau collectée est repartie sur la superficie totale de l'excavation de l'ouvrage. Alors que dans les ouvrages DLM, l'eau collectée est concentrée dans les sousouvrages destinés aux poquets des cultures.

\section{Conclusion}

Cette étude a permis d'évaluer les effets des demi-lunes multifonctionnelles sur la production agricole de sorgho en comparaison avec la pratique locale de zai et les demi-lunes conventionnelles. En effet, il ressort de cette étude que l'installation des nouveaux ouvrages de demi-lunes multifonctionnelles a permis de restaurer les terres dégradées et permettre la production du sorgho dans la région sahélienne de Tahoua au Niger. Ainsi, tout comme les demi-lunes conventionnelles et les zai, les nouveaux ouvrages 
multifonctionnels ont permis la croissance et le développement des cultures du sorgho. La croissance de ces cultures de sorgho-est significativement plus élevée dans les demi-lunes multifonctionnelles que dans les demi-lunes conventionnelles et les zaï après deux campagnes (2019-2020). En outre, le rendement en grains du sorgho est plus élevé dans les DLM (639-2159 kg/ha) que dans les DLC (497-1858 kg/ha) et dans les zaï (189-691 kg/ha) sur la même période que précédemment. Les résultats de cette étude permettent de dire que les demi-lunes multifonctionnelles, tout comme les demi-lunes conventionnelles, sont adaptées au contexte local sahélien du département de Tahoua au Niger et permettent d'augmenter la production agricole de manière significative. Toutefois, il serait nécessaire d'évaluer les impacts de ces ouvrages sur la production agricole à long terme tout en intégrant également d'autres cultures.

\section{References:}

1. Ambouta J.M.K., Bouzou M.I. (2004). Expériences de récupération des sols sahéliens dégradés grâce à l'incorporation de doses variables de fumier et d'un hydro rétenteur fertilisant. Sécheresse 15 (1) ,49-55

2. Amede, T., Tarawali, S., and Peden, D.(2011). Zai improves nutrient and water productivity in the Ethiopian highlands. Experimental Agriculture 47 (1): 7-20.

3. Banque mondiale [2016/11/16]. https://www.banquemondiale.org/fr/news/feature/2016/11/14/africasbold-and-ambitious-endeavor-restoring-100m-hectares-of-land-by2030.

4. Biazin, B., Sterk, G., Temesgen, M., Abdulkedir, A., Stroosnijder, L. (2012). Rainwater harvesting and management in rainfed agricultural systems in sub-Saharan Africa-a review. Physics and Chemistry of the Earth, Parts A/B/C, 47, 139-151

5. Bouzou-Moussa 1., Dan Lamso N., 2004. Le «tassa» : une technique de CES bien adaptée aux conditions physiques et socio-économiques des glacis des régions semi-arides (Niger). Revue de Géographie Alpine, 92 : 61-70.

6. Ding, W., Wang, F., Dong, Y., Jin, K., Cong, C., Han, J., Ge, W. (2021). Effects of rainwater harvesting system on soil moisture in rainfed orchards on the Chinese Loess Plateau. Agricultural Water Management 243 (1) : 106-496.

7. Fatondji, D., Martius, C., Bielders, C.L., Koala S.,. Vlek, P.L.G., Zougmore, R. (2009). Decomposition of organic amendment and nutrient release under the zai technique in the Sahel. Nutrient Cycling in Agroecosystems. Nutrient Cycling in Agroecosystems 85 (3): 225239. 
8. Ganry, F. et Thuriès, L. (2017). Intérêt des fumiers pour restaurer la fertilité des sols en zone semi-aride d'Afrique. In : Restauration de la productivité des sols tropicaux et méditerranéens : contribution à l'agroécologie. Roose, Eric (ed.). Marseille : IRD, pp. 179-195.

9. Kokerai L. K. \& Kugedera A. T. (2019). Review: role of in situ Rainwater Harvesting and integrated soil fertility management on small Grain Productivity. International Journal of Agriculture \& Agribusiness 2 (2) : 249-259.

10. Motsi, K. E., Chuma, E., Mukamuri, B. B. (2004). Rainwater harvesting for sustainable agriculture in communal lands of Zimbabwe. Physics and Chemistry of the Earth, Parts A/B/C, 29(1518), 1069-1073.

11. Nyamekye, C., Thiel, M., Schönbrodt-Stitt, S., Zoungrana, B. J. B., \& Amekudzi, L. K. (2018). Soil and Water Conservation in Burkina Faso, West Africa. Sustainability 10 (9) : 31-82.

12. Okou, F.A.Y.; Tente, B.; Bachmann, Y.; Sinsin, B. (2016). Regional erosion riskmapping for decision support: A case study fromWest Africa. Land Use Policy 56: 27-37.

13. Rockström J, Jansson PE, Barron J. (1998). Seasonal rainfall partitioningunder runon and runoff conditions on sandy soil in Niger. On-farm measurements and water balance modelling. Journal of Hydrology, 210 (1-4): 68-92.

14. Roose E. (2004). La gestion conservatoire de l'eau et de la fertilité des sols: une stratégie nouvelle de la lutte antiérosive pour le développement durable. Sécheresse, 15 (1) :5-7.

15. Roose É., Sabir M., Laouina A. (2010). Gestion durable de l'eau et des sols au Maroc. Valorisation des techniques traditionnelles méditerranéennes. Marseille, IRD Éditions, 343 p.

16. Roose E. (2017). Restauration de la productivité des sols tropicaux et méditerranéens. Contribution à Agroécologie. Edition IRD, pp 711.

17. Sawadogo H. (2006). Fertilisation organique et phosphatéeen système de culture zaï en milieu soudano-sahélien du Burkina Faso. Thèse de doctorat : Faculté universitaire des Sciences agronomiques de Gembloux (Belgique).

18. Svendsen, M., Ewing, M., Msangi, S. (2009). Measuring irrigation performance in Africa. IFPRI Discussion Paper, IFPRI, Washington.

19. Tadros, M. J., Al-Mefleh, N. K., Othman, Y. A., \& Al-Assaf, A. (2021). Water harvesting techniques for improving soil water content, and morpho-physiology of pistachio trees under rainfed conditions. Agricultural Water Management $243: 106464$.

20. Wildemeersch, J. C., Timmerman, E., Mazijn, B., Sabiou, M., Ibro, G., Garba, M., \& Cornelis, W. (2015). Assessing the constraints to adopt 
water and soil conservation techniques in Tillaberi, Niger. Land Degradation \& Development 26 (5): 491-501.

21. Yosef, B. A., \& Asmamaw, D. K. (2015). Rainwater harvesting: An option for dry land agriculture in arid and semi-arid Ethiopia. International Journal of Water Resources and Environmental Engineering, 7(2) : 17-28.

22. Zougmoré, R., Jalloh, A., \& Tioro, A. (2014). Climate-smart soil water and nutrient management options in semiarid West Africa: a review of evidence and analysis of stone bunds and zaï techniques. Agriculture \& Food Security 3 (1): 16.

23. Zougmoré, R., Zida, Z., Kambou, N. F. (2003). Role of nutrientamendments in the success of half-moonsoil and water conservation practice in semiarid Burkina Faso. Soil and Tillage Research, 71(2) : 143-149. 